\title{
An Improvement of the Differential Transformation Method and Its Application for Boundary Layer Flow of a Nanofluid
}

\author{
Abdelhalim Ebaid, ${ }^{1}$ Hassan A. El-Arabawy, ${ }^{1,2}$ and Nader Y. Abd Elazem ${ }^{1}$ \\ ${ }^{1}$ Department of Mathematics, Faculty of Science, University of Tabuk, P.O. Box 741, Tabuk 71491, Saudi Arabia \\ ${ }^{2}$ Department of Mathematics, Faculty of Science, Ain Shams University, Egypt \\ Correspondence should be addressed to Abdelhalim Ebaid; halimgamil@yahoo.com
}

Received 4 April 2013; Accepted 22 April 2013

Academic Editor: Jürgen Geiser

Copyright (c) 2013 Abdelhalim Ebaid et al. This is an open access article distributed under the Creative Commons Attribution License, which permits unrestricted use, distribution, and reproduction in any medium, provided the original work is properly cited.

\begin{abstract}
The main feature of the boundary layer flow problems of nanofluids or classical fluids is the inclusion of the boundary conditions at infinity. Such boundary conditions cause difficulties for any of the series methods when applied to solve such a kind of problems. In order to solve these difficulties, the authors usually resort to either Padé approximants or the commercial numerical codes. However, an intensive work is needed to perform the calculations using Padé technique. Due to the importance of the nanofluids flow as a growing field of research and the difficulties caused by using Padé approximants to solve such problems, a suggestion is proposed in this paper to map the semi-infinite domain into a finite one by the help of a transformation. Accordingly, the differential equations governing the fluid flow are transformed into singular differential equations with classical boundary conditions which can be directly solved by using the differential transformation method. The numerical results obtained by using the proposed technique are compared with the available exact solutions, where excellent accuracy is found. The main advantage of the present technique is the complete avoidance of using Padé approximants to treat the infinity boundary conditions.
\end{abstract}

\section{Introduction}

Nanotechnology is an advanced technology, which deals with the synthesis of nanoparticles, processing of the nano materials and their applications. It is well known that $1 \mathrm{~nm}$ (nanometer) $=10^{-9}$ meter. Normally, if the particle sizes are in the $1-100 \mathrm{~nm}$ range, they are generally called nanoparticles. Nanotechnology has been widely used in industry since materials with sizes of nanometers possess unique physical and chemical properties. Nanoscale particle added fluids are called as nanofluid. The term "nanofluid" was first used by Choi [1] to describe a fluid in which nanometer-sized particles are suspended in conventional heat transfer basic fluids. Fluids such as oil, water, and ethylene glycol mixture are poor heat transfer fluids, since the thermal conductivity of these fluids plays important role on the heat transfer coefficient between the heat transfer medium and the heat transfer surface. Numerous methods have been taken to improve the thermal conductivity of these fluids by suspending nano/micro or larger-sized particle materials in liquids.
An innovative technique to improve heat transfer is by using nanoscale particles in the base fluid [1]. Therefore, the effective thermal conductivity of nanofluids is expected to enhance heat transfer compared with conventional heat transfer liquids (Masuda et al. [2]). This phenomenon suggests the possibility of using nanofluids in advanced nuclear systems (Buongiorno and $\mathrm{Hu}$ [3]). Choi et al. [4] showed that the addition of a small amount (less than $1 \%$ by volume) of nanoparticles to conventional heat transfer liquids increased the thermal conductivity of the fluid up to approximately two times. A comprehensive survey of convective transport in nanofluids was made by Buongiorno and $\mathrm{Hu}$ [3] and very recently by Kakaç and Pramuanjaroenkij [5]. It may be also important to mention that a valuable book in nanofluids is published recently by Das et al. [6]. In addition, various interesting results in this regard can be found in [7-17].

Khan and Pop [18] were the first to investigate the boundary-layer flow of a nanofluid past a stretching sheet. The main feature of the boundary layer flow of nanofluids or classical fluid is the inclusion of the boundary conditions at 
infinity. Such conditions cause difficulties for any of the series methods when applied to solve this kind of problems. This because the infinity boundary condition cannot be applied directly to the series solution, where Padé approximants should be established before applying the boundary condition at infinity. Many authors [19-32] have been resorted to either Padé technique or some numerical commercial codes to solve the boundary value problems in unbounded domain. However, Padé technique requires a massive computational work to obtain accurate approximate solutions. Searching for a direct method to treat the boundary condition at infinity has been the main goal of many researchers for a long time to solve boundary value problems in unbounded domain. Such a direct method is proposed in this paper. The main idea is to transform the physical domain from unbounded into bounded through a transformation. Accordingly, a new system arises which is now subject to classical boundary conditions, where the boundary conditions at infinity disappeared as a result of the new transformation. The transformed system can be directly solved by the differential transformation method (DTM) [33-45] without any need to Padé approximants. In this paper, the governing system of ordinary differential equations describing the boundarylayer flow of a nanofluid past a stretching sheet is analyzed through the proposed improved version of the DTM. The main advantage of the present method is that not only it avoids the use of Padé approximants, but also gives the series solution in a straightforward manner.

\section{Basic Equations}

The basic equations of the steady two-dimensional boundary layer flow of a nanofluid past a stretching surface with the linear velocity $u_{w}(x)=a x$, where $a$ is a constant and $x$ is the coordinate measured along the stretching surface, as given by Kuznetsov and Nield [15] and Nield and Kuznetsov [16] and later by Khan and Pop [18], are as follows:

$$
\begin{gathered}
\frac{\partial u}{\partial x}+\frac{\partial v}{\partial y}=0 \\
u \frac{\partial u}{\partial x}+v \frac{\partial u}{\partial y}=-\frac{1}{\rho_{f}} \frac{\partial p}{\partial x}+v\left(\frac{\partial^{2} u}{\partial x^{2}}+\frac{\partial^{2} u}{\partial y^{2}}\right), \\
u \frac{\partial v}{\partial x}+v \frac{\partial v}{\partial y}=-\frac{1}{\rho_{f}} \frac{\partial p}{\partial y}+v\left(\frac{\partial^{2} v}{\partial x^{2}}+\frac{\partial^{2} v}{\partial y^{2}}\right), \\
u \frac{\partial T}{\partial x}+v \frac{\partial T}{\partial y} \\
=\alpha\left(\frac{\partial^{2} T}{\partial x^{2}}+\frac{\partial^{2} T}{\partial y^{2}}\right)+\tau\left[D_{B}\left(\frac{\partial C}{\partial x} \frac{\partial T}{\partial x}+\frac{\partial C}{\partial y} \frac{\partial T}{\partial y}\right)\right. \\
\left.+\frac{D_{T}}{T_{\infty}}\left(\left(\frac{\partial T}{\partial x}\right)^{2}+\left(\frac{\partial T}{\partial y}\right)^{2}\right)\right] \\
u \frac{\partial C}{\partial x}+v \frac{\partial C}{\partial y}=D_{B}\left(\frac{\partial^{2} C}{\partial x^{2}}+\frac{\partial^{2} C}{\partial y^{2}}\right)+\frac{D_{T}}{T_{\infty}}\left(\frac{\partial^{2} T}{\partial x^{2}}+\frac{\partial^{2} T}{\partial y^{2}}\right),
\end{gathered}
$$

subject to the boundary conditions:

$$
\begin{gathered}
v=0, \quad u=u_{w}(x)=a x, \quad T=T_{w}, \quad C=C_{w}, \quad \text { at } y=0, \\
u=v=0, \quad T=T_{\infty}, \quad C=C_{\infty}, \quad \text { as } y \longrightarrow \infty .
\end{gathered}
$$

A complete physical description of the present problem was well presented by Khan and Pop [18] as follows. The flow takes place at $y \geq 0$, where $y$ is the coordinate measured normal to the stretching surface. A steady uniform stress leading to equal and opposite forces is applied along the $x$ axis so that the sheet is stretched keeping the origin fixed. It is assumed that at the stretching surface, the temperature $T$ and the nanoparticle fraction $C$ take constant values $T_{w}$ and $C_{w}$, respectively. The ambient values, attained as $y$ tends to infinity, of $T$ and $C$, are denoted by $T_{\infty}$ and $C_{\infty}$, respectively.

Here $u$ and $v$ are the velocity components along the axes $x$ and $y$, respectively, $p$ is the fluid pressure, $\rho_{f}$ is the density of the base fluid, $\alpha$ is the thermal diffusivity, $v$ is the kinematic viscosity, $a$ is a positive constant, $D_{B}$ is the Brownian diffusion coefficient, $D_{T}$ is the thermophoretic diffusion coefficient, $\tau=(\rho c)_{p} /(\rho c)_{f}$ is the ratio between the effective heat capacity of the nanoparticle material and heat capacity of the fluid with $\rho$ being the density, $c$ is the volumetric volume expansion coefficient, and $\rho_{p}$ is the density of the particles. Khan and Pop [18] have looked for a similarity solution of (1) with the boundary conditions (2) by assuming that

$$
\begin{gathered}
\psi=(a v)^{1 / 2} x f(\eta), \quad \theta(\eta)=\frac{T-T_{\infty}}{T_{w}-T_{\infty}}, \\
\phi(\eta)=\frac{C-C_{\infty}}{C_{w}-C_{\infty}}, \quad \eta=\left(\frac{a}{\nu}\right)^{1 / 2} y,
\end{gathered}
$$

where the stream function $\psi$ is defined in the usual way as $u=$ $\partial \psi / \partial y$ and $v=-\partial \psi / \partial x$. Hence, a set of ordinary differential equations were obtained by [18] as

$$
\begin{gathered}
f^{\prime \prime \prime}+f f^{\prime \prime}-\left(f^{\prime}\right)^{2}=0, \\
\frac{1}{\operatorname{Pr}} \theta^{\prime \prime}+f \theta^{\prime}+\mathrm{Nb} \phi^{\prime} \theta^{\prime}+\mathrm{Nt}\left(\theta^{\prime}\right)^{2}=0, \\
\phi^{\prime \prime}+\operatorname{Le} f \phi^{\prime}+\frac{\mathrm{Nt}}{\mathrm{Nb}} \theta^{\prime \prime}=0,
\end{gathered}
$$

subject to the boundary conditions:

$$
\begin{gathered}
f(0)=0, \quad f^{\prime}(0)=1, \quad \theta(0)=1, \quad \phi(0)=1, \\
f^{\prime}(\infty)=0, \quad \theta(\infty)=0, \quad \phi(\infty)=0,
\end{gathered}
$$

where primes denote differentiation with respect to $\eta$ and the four parameters are defined by

$$
\begin{aligned}
\operatorname{Pr}=\frac{\nu}{\alpha}, \quad \mathrm{Le} & =\frac{\nu}{D_{B}}, \quad \mathrm{Nb}=\frac{(\rho c)_{p} D_{B}\left(\phi_{w}-\phi_{\infty}\right)}{(\rho c)_{f} \nu}, \\
\mathrm{Nt} & =\frac{(\rho c)_{p} D_{T}\left(T_{w}-T_{\infty}\right)}{(\rho c)_{f} \nu T_{\infty}},
\end{aligned}
$$


where $\mathrm{Pr}, \mathrm{Le}, \mathrm{Nb}$, and $\mathrm{Nt}$ denote the Prandtl number, the Lewis number, the Brownian motion parameter, and the thermophoresis parameter, respectively. The quantities of practical interest are the Nusselt number $\mathrm{Nu}$ and the Sherwood number Sh which are defined as

$$
\begin{gathered}
\mathrm{Nu}=\frac{x q_{w}}{k\left(T_{w}-T_{\infty}\right)}, \\
\mathrm{Sh}=\frac{x q_{m}}{D_{B}\left(C_{w}-C_{\infty}\right)},
\end{gathered}
$$

where $q_{w}$ and $q_{m}$ are the wall heat and mass fluxes, respectively. According to Kuznetsov and Nield [15], $\operatorname{Re}_{x}^{-1 / 2} \mathrm{Nu}$ and $\operatorname{Re}_{x}^{-1 / 2}$ Sh are known as the reduced Nusselt number Nur and reduced Sherwood number Shr, respectively,

$$
\begin{aligned}
& \text { Nur }=\operatorname{Re}_{x}^{-1 / 2} \mathrm{Nu}=-\theta^{\prime}(0), \\
& \text { Shr }=\operatorname{Re}_{x}^{-1 / 2} \mathrm{Sh}=-\phi^{\prime}(0),
\end{aligned}
$$

where $\operatorname{Re}_{x}=x u_{w}(x) / v$ is the local Reynolds number based on the stretching velocity $u_{w}(x)$. It should be noted that the exact solution of (4) with the boundary conditions given in (7) was first obtained by Crane [46] and given as

$$
f(\eta)=1-e^{-\eta} \text {. }
$$

Substituting $f(\eta)$ into (5)-(6), the given system reduces to a system of two coupled differential equations as

$$
\begin{gathered}
\theta^{\prime \prime}+\operatorname{Pr}\left(1-e^{-\eta}+\operatorname{Nb} \phi^{\prime}\right) \theta^{\prime}+\mathrm{Nt}\left(\theta^{\prime}\right)^{2}=0, \\
\phi^{\prime \prime}+\operatorname{Le}\left(1-e^{-\eta}\right) \phi^{\prime}+\frac{\mathrm{Nt}}{\mathrm{Nb}} \theta^{\prime \prime}=0,
\end{gathered}
$$

subject to the boundary conditions:

$$
\begin{array}{ll}
\theta(0)=1, & \theta(\infty)=0, \\
\phi(0)=1, & \phi(\infty)=0 .
\end{array}
$$

\section{Transformed Equations}

In order to solve boundary value problems in unbounded domain by using the DTM, authors are usually resort to Padé approximant due to the boundary condition at infinity, and an approximate solution is only available in this case. As a well-known fact, Padé approximant requires a huge amount of computational work to find out the approximate solution. In this regard, we think that if it is possible to transform the unbounded domain into a bounded one then the BVPs may be easily solved without any need to Padé approximant. A first step in this direction is to transform the unbounded domain of the independent variable $\eta \in[0, \infty)$ into a bounded one $t \in[0,1)$. Such a transformation is found as $t=1-e^{-\eta}$, accordingly the governing equations should be changed to be in terms of the new variable $t$. The effectiveness of this procedure shall be discussed in the next subsection to show the possibility of obtaining very accurate numerical solutions. In view of the mentioned transformation, the system (1)-(3) with the boundary conditions (7) is transformed into a new system in bounded domain given by

$$
\begin{aligned}
& (1-t)^{2} \theta^{\prime \prime}-(1-t)(1-\operatorname{Pr} t) \theta^{\prime} \\
& +\operatorname{Pr}(1-t)^{2}\left[\operatorname{Nb} \theta^{\prime} \phi^{\prime}+\mathrm{Nt}\left(\theta^{\prime}\right)^{2}\right]=0, \\
& (1-t)^{2} \phi^{\prime \prime}-(1-t)(1-\text { Le } t) \phi^{\prime} \\
& +\frac{\mathrm{Nt}}{\mathrm{Nb}}\left[(1-t)^{2} \theta^{\prime \prime}-(1-t) \theta^{\prime}\right]=0,
\end{aligned}
$$

with the boundary conditions:

$$
\begin{array}{ll}
\theta(0)=1, & \theta(1)=0, \\
\phi(0)=1, & \phi(1)=0,
\end{array}
$$

where primes denote differentiation with respect to $t$.

\section{Analysis and Results}

In this section, the application of the DTM is discussed without resorting to Padé approximants. Applying the DTM to the previously mentioned system yielded the following recurrence scheme:

$$
\begin{aligned}
& \sum_{m=0}^{k}(k-m+1)(k-m+2)[\delta(m)-\delta(m-1)] \Theta(k-m+2) \\
& -\sum_{m=0}^{k}(k-m+1) \times[\delta(m)-\operatorname{Pr} \delta(m-1)] \Theta(k-m+1) \\
& +\mathrm{Nb} \operatorname{Pr} \sum_{r=0}^{k} \sum_{m=0}^{r}(m+1)(r-m+1) \\
& \times[\delta(k-r)-\delta(k-r-1)] \Theta(m+1) \\
& \times \Phi(r-m+1) \\
& +\mathrm{Nt} \operatorname{Pr} \sum_{r=0}^{k} \sum_{m=0}^{r}(m+1)(r-m+1)[\delta(k-r)-\delta(k-r-1)] \\
& \times \Theta(m+1) \Theta(r-m+1)=0, \\
& \sum_{m=0}^{k}(k-m+1)(k-m+2)[\delta(m)-\delta(m-1)] \Phi(k-m+2) \\
& -\sum_{m=0}^{k}(k-m+1) \times[\delta(m)-\operatorname{Le} \delta(m-1)] \Phi(k-m+1) \\
& +\frac{\mathrm{Nt}}{\mathrm{Nb}}\left(\sum_{m=0}^{k}(k-m+1)(k-m+2)[\delta(m)-\delta(m-1)]\right. \\
& \times \Theta(k-m+2)-(k+1) \Theta(k+1))=0,
\end{aligned}
$$


with the transformed boundary conditions

$$
\begin{array}{ll}
\Theta(0)=1, & \sum_{k=0}^{N} \Theta(k)=0, \\
\Phi(0)=1, & \sum_{k=0}^{N} \Phi(k)=0 .
\end{array}
$$

Equations (17a), (17b), (18), and (19) are used to obtain very accurate approximate numerical solutions, where two different cases are derived and discussed in the next two subsections.

4.1. Case 1: At $N t=0$ and $N b=0$. At $\mathrm{Nt}=0$ and $\mathrm{Nb}=0$, the boundary value problem for $\phi$ becomes ill-posed and consequently the boundary value problem for $\theta$ becomes

$$
(1-t)^{2} \theta^{\prime \prime}-(1-t)(1-\operatorname{Pr} t) \theta^{\prime}=0,
$$

subject to the boundary conditions in (15). Accordingly, a simple recurrence scheme is obtained form (17a) and (17b), where $\mathrm{Nt}=\mathrm{Nb}=0$ are inserted, hence

$$
\begin{aligned}
\sum_{m=0}^{k}(k-m+1) & \\
\times( & (k-m+2)[\delta(m)-\delta(m-1)] \Theta(k-m+2) \\
- & {[\delta(m)-\operatorname{Pr} \delta(m-1)] \Theta(k-m+1)) . }
\end{aligned}
$$

Using the recurrence scheme (21) with the transformed initial conditions (18) for $k=0,1,2, \ldots, 8$, a system of algebraic equations is obtained in $\Theta(1), \Theta(2), \ldots$, and $\Theta(10)$. Solving this system, the 10-term approximate solution at any Prandtl number is given in terms of the original similarity variable $\eta$ as

$$
\begin{aligned}
& \Theta_{10}(\eta) \\
& =1+\Delta\left[-3628800\left(1-e^{-\eta}\right)-1814400\left(1-e^{-\eta}\right)^{2}\right. \\
& +604800(\operatorname{Pr}-2)\left(1-e^{-\eta}\right)^{3} \\
& +151200(5 \operatorname{Pr}-6)\left(1-e^{-\eta}\right)^{4} \\
& -30240\left(3 \operatorname{Pr}^{2}-26 \operatorname{Pr}+24\right)\left(1-e^{-\eta}\right)^{5} \\
& -5040\left(35 \operatorname{Pr}^{2}-154 \operatorname{Pr}+120\right)\left(1-e^{-\eta}\right)^{6} \\
& +720\left(15 \operatorname{Pr}^{3}-340 \operatorname{Pr}^{2}+1044 \operatorname{Pr}-720\right) \\
& \times\left(1-e^{-\eta}\right)^{7} \\
& +90\left(315 \operatorname{Pr}^{3}-3304 \operatorname{Pr}^{2}+8028 \operatorname{Pr}-5040\right) \\
& \times\left(1-e^{-\eta}\right)^{8} \\
& -10\left(105 \operatorname{Pr}^{4}-4900 \operatorname{Pr}^{3}+33740 \operatorname{Pr}^{2}\right. \\
& -69264 \operatorname{Pr}+40320)\left(1-e^{-\eta}\right)^{9}
\end{aligned}
$$

$$
\begin{gathered}
-\left(3465 \operatorname{Pr}^{4}-70532 \operatorname{Pr}^{3}+367884 \operatorname{Pr}^{2}\right. \\
\left.-663696 \operatorname{Pr}+362880)\left(1-e^{-\eta}\right)^{10}\right],
\end{gathered}
$$

where

$$
\Delta=\frac{1}{4515^{4}-158682^{3}+1514564^{2}-5753736+10628640} .
$$

Here, it may be useful to mention that an exact solution for the current case is obtained very recently by the first author in [47] and given by

$$
\theta(\eta)=\frac{\Gamma\left(\operatorname{Pr}, 0, \operatorname{Pr} e^{-\eta}\right)}{\Gamma(\operatorname{Pr}, 0, \operatorname{Pr})}
$$

where $\Gamma\left(a, z_{0}, z_{1}\right)=\int_{z_{0}}^{z_{1}} \mu^{a-1} e^{-\mu} d \mu$ is the generalized Gamma function which can be expressed in terms of the incomplete Gamma function as $\Gamma\left(a, z_{0}, z_{1}\right)=\Gamma\left(a, z_{0}\right)-\Gamma\left(a, z_{1}\right)$, where $\Gamma(a, z)=\int_{z}^{\infty} \mu^{a-1} e^{-\mu} d \mu$. There is no doubt that the availability of the exact solution gives the opportunity to validate the accuracy of the suggested approach. For the purpose of illustration, the approximate solutions are compared in Figures 1, 2, and 3 with the exact one given by (24) at different values of Prandtl number. These primary results reveal that the 10-term approximate solution is sufficient to obtain numerical solutions of high accuracy for certain range of Prandtl number, mainly $\operatorname{Pr}=1,2,3$. However, at $\operatorname{Pr}=4$ the 10-term approximate solution is not accurate as can be seen in Figure 4. This observation leads to the conclusion that with increasing $\mathrm{Pr}$ more terms in the approximate series solution are in fact needed. For example, at $\operatorname{Pr}=5$ the 20term approximate solution is found sufficient in Figure 5, while 30-term approximate solution is found identical to the exact one at $\operatorname{Pr}=10$ in Figure 6. However, the main advantage of the suggested approach is the avoidance of Padéapproximants which has been used a long time to treat the boundary condition at infinity.

4.2. Case 2: At $N t=0, \operatorname{Pr}=L e=1$, and $N b \neq 0$. Substituting $\mathrm{Nt}=0, \operatorname{Pr}=1$, and Le $=1$ into (17a) and (17b) yields

$$
\begin{aligned}
& \sum_{m=0}^{k}(k-m+1)(k-m+2)[\delta(m)-\delta(m-1)] \Theta(k-m+2) \\
& -\sum_{m=0}^{k}(k-m+1) \times[\delta(m)-\operatorname{Pr} \delta(m-1)] \Theta(k-m+1) \\
& +\operatorname{Nb} \operatorname{Pr} \sum_{r=0}^{k} \sum_{m=0}^{r}(m+1)(r-m+1) \\
& \times[\delta(k-r)-\delta(k-r-1)] \Theta(m+1) \\
& \times \Phi(r-m+1)=0
\end{aligned}
$$




$$
\begin{aligned}
& \sum_{m=0}^{k}(k-m+1)(k-m+2)[\delta(m)-\delta(m-1)] \Phi(k-m+2) \\
& -\sum_{m=0}^{k}(k-m+1) \times[\delta(m)-\operatorname{Le} \delta(m-1)] \\
& \quad \times \Phi(k-m+1)=0 .
\end{aligned}
$$

Using the recurrence scheme (25) with the transformed initial conditions (18) and (19) for $k=0,1,2, \ldots, 4$, we get a system of algebraic equations in $\Theta(1), \Theta(2), \ldots, \Theta(6)$ and $\Phi(1), \Phi(2), \ldots, \Phi(6)$. The solution of the required system leads to the following 6-term approximate solution for the $\theta$ equation:

$$
\begin{aligned}
\Theta_{6}(\eta)= & 1+\Theta(1)\left(1-e^{-\eta}\right)+\Theta(2)\left(1-e^{-\eta}\right)^{2} \\
& +\Theta(3)\left(1-e^{-\eta}\right)^{3}+\Theta(4)\left(1-e^{-\eta}\right)^{4} \\
& +\Theta(5)\left(1-e^{-\eta}\right)^{5}+\Theta(6)\left(1-e^{-\eta}\right)^{6}
\end{aligned}
$$

where $\Theta(1), \Theta(2), \ldots$, and $\Theta(6)$ are expressed in terms of $\mathrm{Nb}$ but ignored here for lengthy results. However, the 6-term series solution for the $\phi$ equation is given explicitly as

$$
\begin{aligned}
\Phi_{6}(\eta) & \\
=1 & \frac{1}{1237} \\
\times & {\left[720\left(1-e^{-\eta}\right)+360\left(1-e^{-\eta}\right)^{2}+120\left(1-e^{-\eta}\right)^{3}\right.} \\
& \left.+30\left(1-e^{-\eta}\right)^{4}+6\left(1-e^{-\eta}\right)^{5}+\left(1-e^{-\eta}\right)^{6}\right] .
\end{aligned}
$$

The exact solutions are obtained in [47] as

$$
\begin{gathered}
\phi(\eta)=\frac{1-e^{-e^{-\eta}}}{1-e^{-1}}, \\
\theta(\eta)=\frac{1-e^{-\alpha \mathrm{Nb}\left(1-e^{-e^{-\eta}}\right)}}{1-e^{-\mathrm{Nb}}} .
\end{gathered}
$$

The obtained truncated series solution $\Theta_{6}(\eta)$ is compared with the exact one in Figures 7-9 at several values of $\mathrm{Nb}$. As observed from Figures 7 and 8, the approximate solution is coincided with the exact one at certain values, $\mathrm{Nb}=0.1$ and $\mathrm{Nb}=0.3$. However, it approaches the exact curve at $\mathrm{Nb}=0.5$, where more terms are needed in this case. In addition, the approximate solution $\Phi_{6}(\eta)$ is found identical to the exact curve as shown in Figure 10.

\section{Conclusions}

A system of ordinary differential equations describing the boundary layer flow of a nanofluid past a stretching sheet is investigated in this paper via a new approach. The suggested approach is based on transforming the boundary conditions

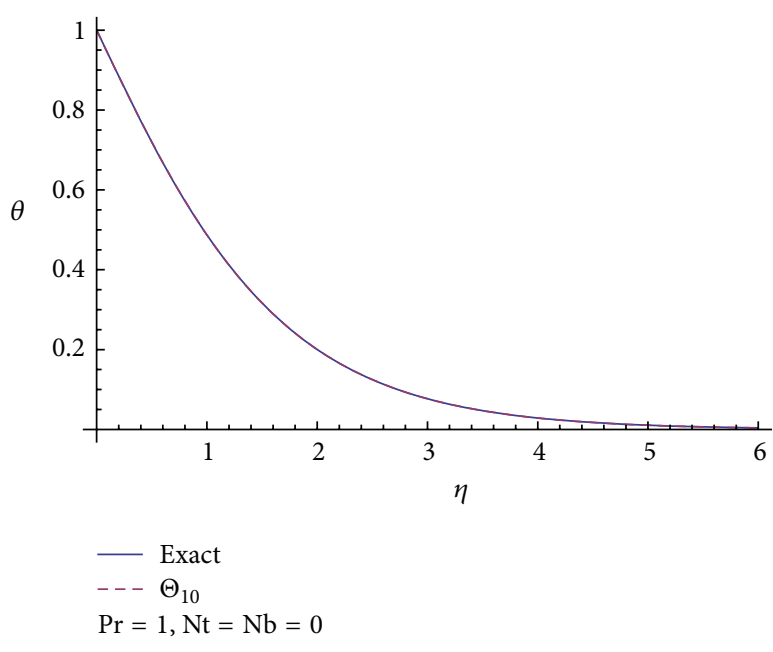

Figure 1

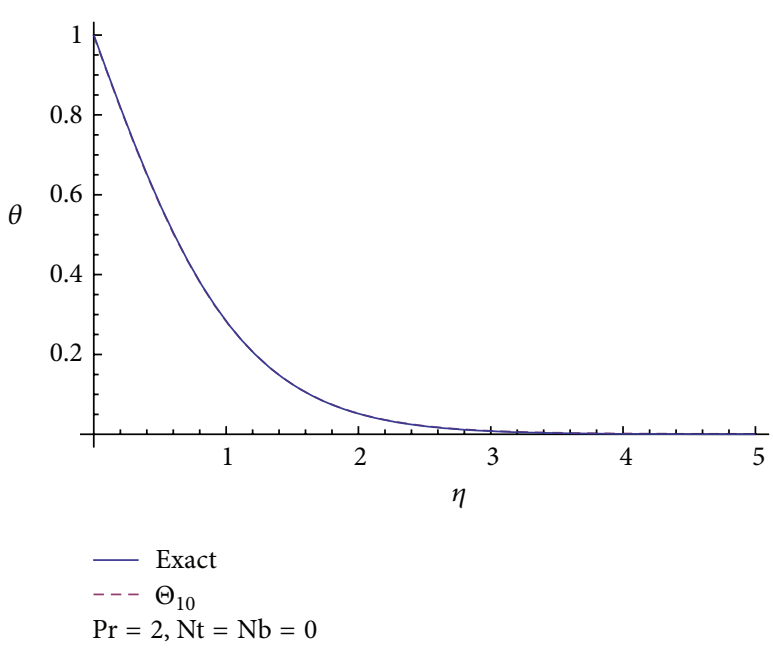

FIGURE 2

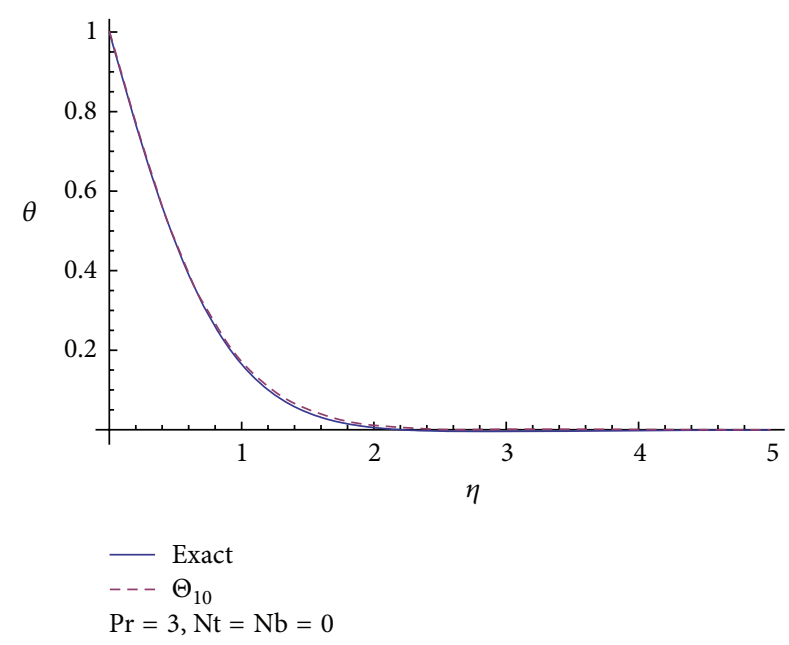

FIGURE 3 

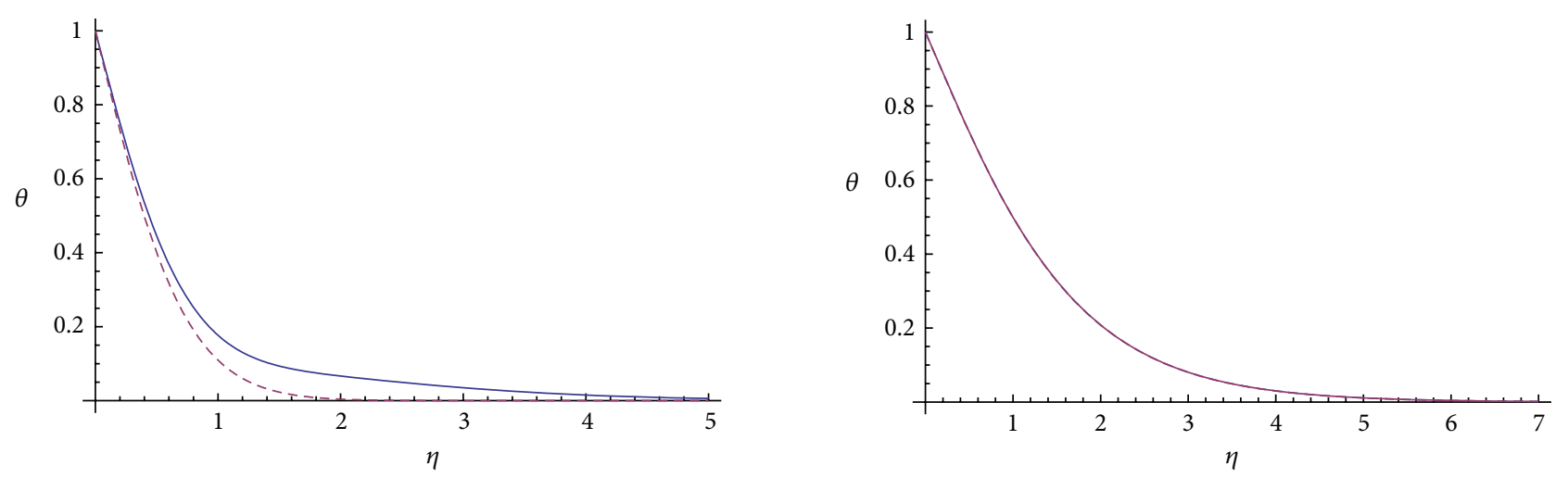

$$
\begin{aligned}
& \text { Exact } \\
& ---\Theta_{10} \\
& \operatorname{Pr}=4, \mathrm{Nt}=\mathrm{Nb}=0
\end{aligned}
$$$$
\text { - Exact }
$$$$
\text { - - - } \Theta_{6}
$$$$
\mathrm{Pr}=\mathrm{Le}=1, \mathrm{Nt}=0, \mathrm{Nb}=0.1
$$

Figure 4

Figure 7
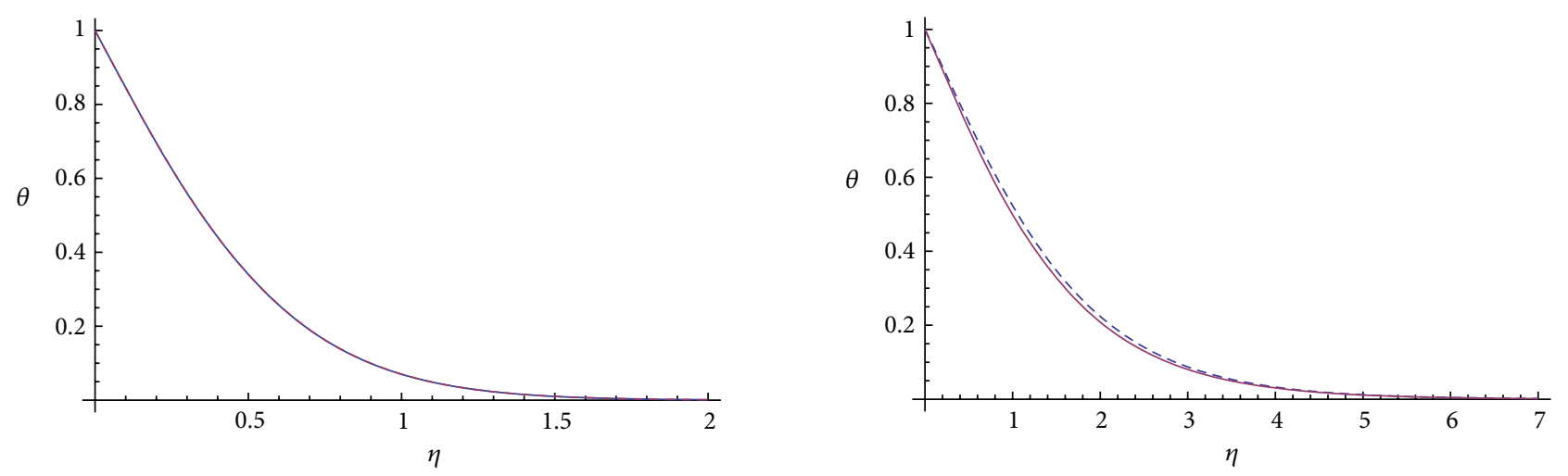

- Exact

- - $\Theta_{20}$

$\mathrm{Pr}=5, \mathrm{Nt}=\mathrm{Nb}=0$

FIgURe 5

$$
\begin{aligned}
& \text { Exact } \\
& --\Theta_{6} \\
& \operatorname{Pr}=\mathrm{Le}=1, \mathrm{Nt}=0, \mathrm{Nb}=0.3
\end{aligned}
$$

Figure 8
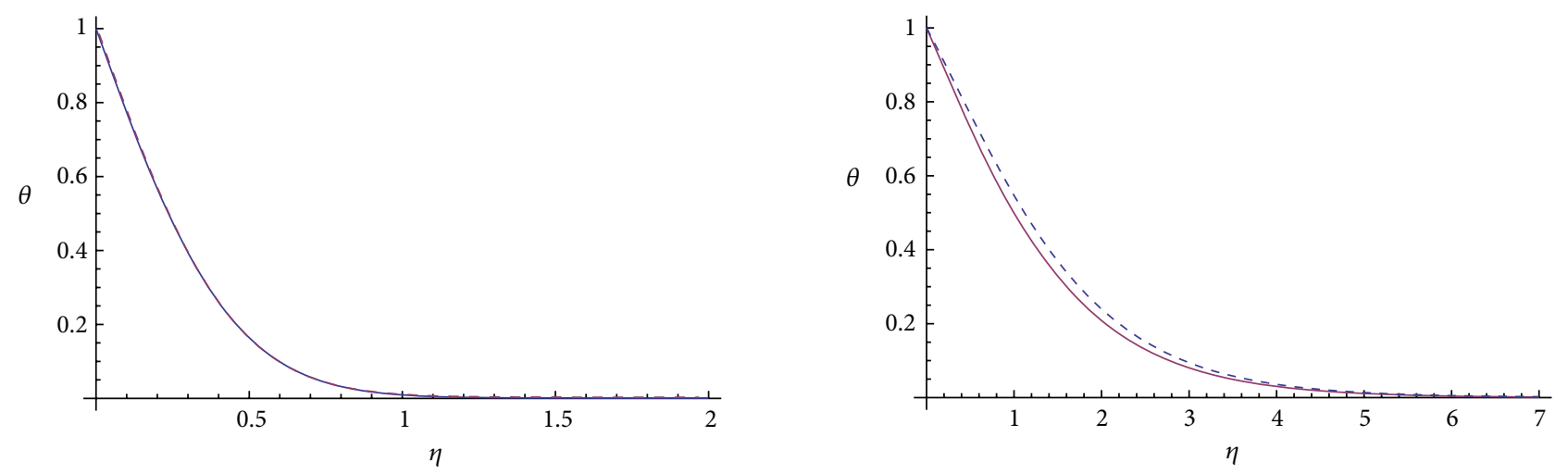

- Exact

$---\Theta_{30}$

$\mathrm{Pr}=10, \mathrm{Nt}=\mathrm{Nb}=0$

$$
\begin{aligned}
& \text { Exact } \\
& ---\Theta_{6} \\
& \operatorname{Pr}=\mathrm{Le}=1, \mathrm{Nt}=0, \mathrm{Nb}=0.5
\end{aligned}
$$

FIGURE 6 


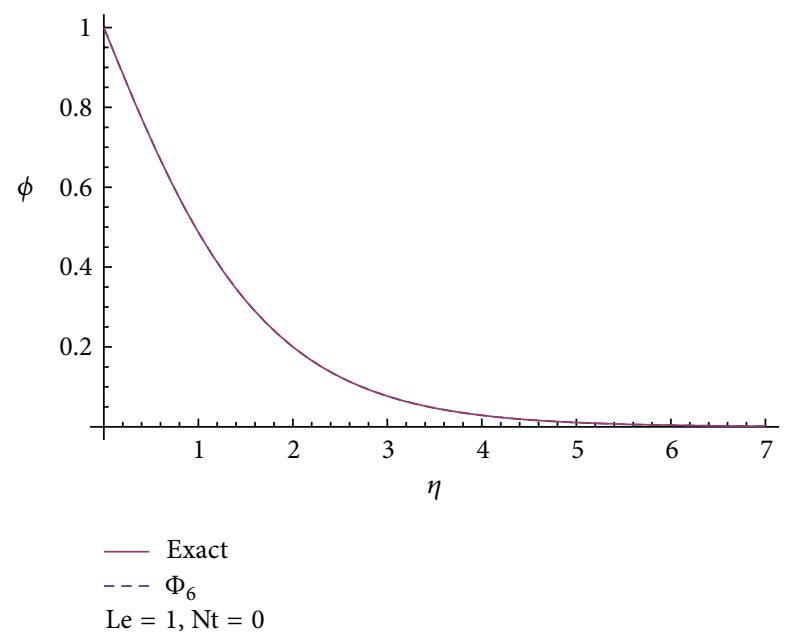

FIGURE 10

at infinity into classical conditions prior to the application of the differential transformation method. A transformation is successfully used to map the unbounded physical domain into a bounded one. In addition, the current results are validated through various comparisons with the available exact solutions. In comparison with Padé technique, the new method of solution is found not only straightforward but also effective in obtaining accurate numerical solutions, where Padé approximant was completely avoided.

\section{Acknowledgments}

This paper was funded by the Deanship of Scientific Research (DSR), University of Tabuk, Tabuk, under Grant no. (S-841432). The authors, therefore, acknowledge with thanks DSR technical and financial support.

\section{References}

[1] S. U. S. Choi, "Enhancing thermal conductivity of fluids with nanoparticles," in The Proceedings of the ASME International Mechanical Engineering Congress and Exposition, ASME, FED 231/MD 66, pp. 99-105, San Francisco, Calif, USA, 1995.

[2] H. Masuda, A. Ebata, K. Teramae, and N. Hishinuma, "Alterlation of thermal conductivity and viscosity of liquid by dispersing ultra-fine particles (Dispersion of $\mathrm{g}-\mathrm{Al}_{2} \mathrm{O}_{3}, \mathrm{SiO}_{2}$, and $\mathrm{TiO}_{2}$ ultra-fine particles)," Netsu Bussei, vol. 7, no. 4, pp. 227233, 1993.

[3] J. Buongiorno and W. Hu, "Nanofluid coolants for advanced nuclear power plants," in Proceedings of ICAPP '05, Paper no. 5705, Seoul, Republic of Korea, May 2005.

[4] S. U. S. Choi, Z. G. Zhang, W. Yu, F. E. Lockwood, and E. A. Grulke, "Anomalous thermal conductivity enhancement in nanotube suspensions," Applied Physics Letters, vol. 79, no. 14, pp. 2252-2254, 2001.

[5] S. Kakaç and A. Pramuanjaroenkij, "Review of convective heat transfer enhancement with nanofluids," International Journal of Heat and Mass Transfer, vol. 52, no. 13-14, pp. 3187-3196, 2009.

[6] S. K. Das, S. U. S. Choi, W. Yu, and T. Pradeep, Nanofluids: Science and Technology, Wiley-Interscience, Hoboken, NJ, USA, 2007.
[7] J. Buongiorno, "Convective transport in nanofluids," Journal of Heat Transfer, vol. 128, no. 3, pp. 240-250, 2006.

[8] D. A. Nield and A. V. Kuznetsov, "The Cheng-Minkowycz problem for natural convective boundary-layer flow in a porous medium saturated by a nanofluid," International Journal of Heat and Mass Transfer, vol. 52, no. 25-26, pp. 5792-5795, 2009.

[9] K. Khanafer, K. Vafai, and M. Lightstone, "Buoyancy-driven heat transfer enhancement in a two-dimensional enclosure utilizing nanofluids," International Journal of Heat and Mass Transfer, vol. 46, no. 19, pp. 3639-3653, 2003.

[10] W. Daungthongsuk and S. Wongwises, "A critical review of convective heat transfer of nanofluids," Renewable and Sustainable Energy Reviews, vol. 11, no. 5, pp. 797-817, 2007.

[11] R. K. Tiwari and M. K. Das, "Heat transfer augmentation in a two-sided lid-driven differentially heated square cavity utilizing nanofluids," International Journal of Heat and Mass Transfer, vol. 50, no. 9-10, pp. 2002-2018, 2007.

[12] L. Wang and X. Wei, "Heat conduction in nanofluids," Chaos, Solitons and Fractals, vol. 39, no. 5, pp. 2211-2215, 2009.

[13] H. F. Oztop and E. Abu-Nada, "Numerical study of natural convection in partially heated rectangular enclosures filled with nanofluids," International Journal of Heat and Fluid Flow, vol. 29, no. 5, pp. 1326-1336, 2008.

[14] A. V. Kuznetsov and D. A. Nield, "Natural convective boundarylayer flow of a nanofluid past a vertical plate," International Journal of Thermal Sciences, vol. 49, no. 2, pp. 243-247, 2010.

[15] A. V. Kuznetsov and D. A. Nield, "Effect of local thermal nonequilibrium on the onset of convection in a porous medium layer saturated by a nanofluid," Transport in Porous Media, vol. 83, no. 2, pp. 425-436, 2010.

[16] E. H. Aly and A. Ebaid, "New exact solutions for boundarylayer flow of a nanofluid past a stretching sheet," Journal of Computational and Theoretical Nanoscience. In press.

[17] P. Cheng and W. J. Minkowycz, "Free convection about a vertical flat plate embedded in a porous medium with application to heat transfer from a dike," Journal of Geophysical Research, vol. 82, no. 14, pp. 2040-2044, 1977.

[18] W. A. Khan and I. Pop, "Boundary-layer flow of a nanofluid past a stretching sheet," International Journal of Heat and Mass Transfer, vol. 53, no. 11-12, pp. 2477-2483, 2010.

[19] J. P. Boyd, "Padé-approximant algorithm for solving nonlinear ordinary differential equation boundary value problems on an unbounded domain," Computers in Physics, vol. 11, no. 3, pp. 299-303, 1997.

[20] A. M. Wazwaz, "The modified decomposition method and Padé approximants for solving the Thomas-Fermi equation," Applied Mathematics and Computation, vol. 105, no. 1, pp. 11-19, 1999.

[21] A. M. Wazwaz, "The modified decomposition method and Padé approximants for a boundary layer equation in unbounded domain," Applied Mathematics and Computation, vol. 177, no. 2, pp. 737-744, 2006.

[22] A. M. Wazwaz, "Padé approximants and Adomian decomposition method for solving the Flierl-Petviashivili equation and its variants," Applied Mathematics and Computation, vol. 182, no. 2, pp. 1812-1818, 2006.

[23] A. M. Wazwaz, "The variational iteration method for solving two forms of Blasius equation on a half-infinite domain," Applied Mathematics and Computation, vol. 188, no. 1, pp. 485-491, 2007. 
[24] S. A. Kechil and I. Hashim, "Non-perturbative solution of freeconvective boundary-layer equation by Adomian decomposition method," Physics Letters A, vol. 363, no. 1-2, pp. 110-114, 2007.

[25] S. A. Kechil and I. Hashim, "Series solution of flow over nonlinearly stretching sheet with chemical reaction and magnetic field," Physics Letters A, vol. 372, no. 13, pp. 2258-2263, 2008.

[26] E. Alizadeh, K. Sedighi, M. Farhadi, and H. R. Ebrahimi-Kebria, "Analytical approximate solution of the cooling problem by Adomian decomposition method," Communications in Nonlinear Science and Numerical Simulation, vol. 14, no. 2, pp. 462-472, 2009.

[27] T. Hayat, Q. Hussain, and T. Javed, "The modified decomposition method and Padé approximants for the MHD flow over a non-linear stretching sheet," Nonlinear Analysis: Real World Applications, vol. 10, no. 2, pp. 966-973, 2009.

[28] S. Abbasbandy and T. Hayat, "Solution of the MHD FalknerSkan flow by Hankel-Padé method," Physics Letters A, vol. 373, no. 7, pp. 731-734, 2009.

[29] M. M. Rashidi and E. Erfani, "A novel analytical solution of the thermal boundary-layer over a flat plate with a convective surface boundary condition using DTM-Padé," in Proceedings of the International Conference on Signal Processing Systems (ICSPS '09), pp. 905-909, Singapore, May 2009.

[30] M. M. Rashidi and S. A. M. Pour, "A novel analytical solution of steady flow over a rotating disk in porous medium with heat transfer by DTM-Padé," African Journal of Mathematics and Computer Science Research, vol. 3, no. 6, pp. 93-100, 2010.

[31] M. M. Rashidi and S. A. M. Pour, "Explicit solution of axisymmetric stagnation flow towards a shrinking sheet by DTMPadé," Applied Mathematical Sciences, vol. 4, no. 53-56, pp. 2617-2632, 2010.

[32] M. M. Rashidi and M. Keimanesh, "Using differential transform method and Padé approximant for solving mhd flow in a laminar liquid film from a horizontal stretching surface," Mathematical Problems in Engineering, vol. 2010, Article ID 491319, 14 pages, 2010.

[33] J. K. Zhou, Differential Transformation and Its Applications for Electrical circuIts, Huazhong University Press, Wuhan, China, 1986.

[34] S. H. Ho and C. K. Chen, "Analysis of general elastically end restrained non-uniform beams using differential transform," Applied Mathematical Modelling, vol. 22, no. 4-5, pp. 219-234, 1998.

[35] C. K. Chen and S. H. Ho, “Transverse vibration of a rotating twisted Timoshenko beams under axial loading using differential transform," International Journal of Mechanical Sciences, vol. 41, no. 11, pp. 1339-1356, 1999.

[36] C. K. Chen and S. H. Ho, "Solving partial differential equations by two-dimensional differential transform method," Applied Mathematics and Computation, vol. 106, no. 2-3, pp. 171-179, 1999.

[37] M. J. Jang, C. L. Chen, and Y. C. Liy, "On solving the initialvalue problems using the differential transformation method," Applied Mathematics and Computation, vol. 115, no. 2-3, pp. 145$160,2000$.

[38] M. J. Jang, C. L. Chen, and Y. C. Liu, “Two-dimensional differential transform for partial differential equations," Applied Mathematics and Computation, vol. 121, no. 2-3, pp. 261-270, 2001.
[39] M. Köksal and S. Herdem, "Analysis of nonlinear circuits by using differential Taylor transform," Computers and Electrical Engineering, vol. 28, no. 6, pp. 513-525, 2002.

[40] F. Ayaz, "Solutions of the system of differential equations by differential transform method," Applied Mathematics and Computation, vol. 147, no. 2, pp. 547-567, 2004.

[41] A. Arikoglu and I. Ozkol, "Solution of boundary value problems for integro-differential equations by using differential transform method," Applied Mathematics and Computation, vol. 168, no. 2, pp. 1145-1158, 2005.

[42] S. H. Chang and I. L. Chang, "A new algorithm for calculating one-dimensional differential transform of nonlinear functions," Applied Mathematics and Computation, vol. 195, no. 2, pp. 799808, 2008.

[43] A. S. V. R. Kanth and K. Aruna, "Two-dimensional differential transform method for solving linear and non-linear Schrödinger equations," Chaos, Solitons and Fractals, vol. 41, no. 5, pp. 2277-2281, 2009.

[44] A. S. V. R. Kanth and K. Aruna, "Differential transform method for solving the linear and nonlinear Klein-Gordon equation," Computer Physics Communications, vol. 180, no. 5, pp. 708-711, 2009.

[45] A. Ebaid, "Approximate periodic solutions for the non-linear relativistic harmonic oscillator via differential transformation method," Communications in Nonlinear Science and Numerical Simulation, vol. 15, no. 7, pp. 1921-1927, 2010.

[46] L. J. Crane, "Flow past a stretching plate," Zeitschrift für Angewandte Mathematik und Physik, vol. 21, no. 4, pp. 645-647, 1970.

[47] A. Ebaid and M. D. Aljoufi, "New theoretical and numerical results for the boundary-layer flow of a nanofluid past a stretching sheet," Advanced Studies in Theoretical Physics. In press. 


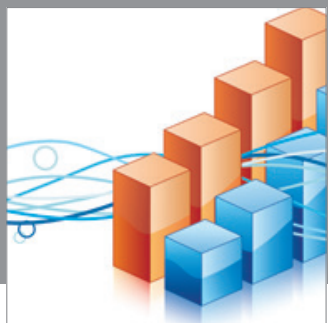

Advances in

Operations Research

mansans

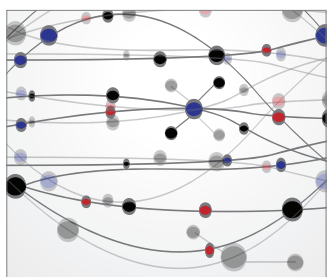

The Scientific World Journal
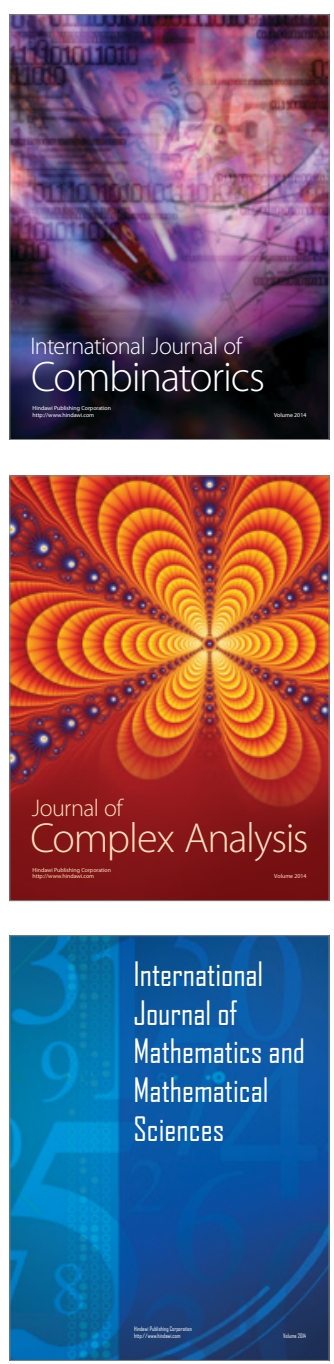
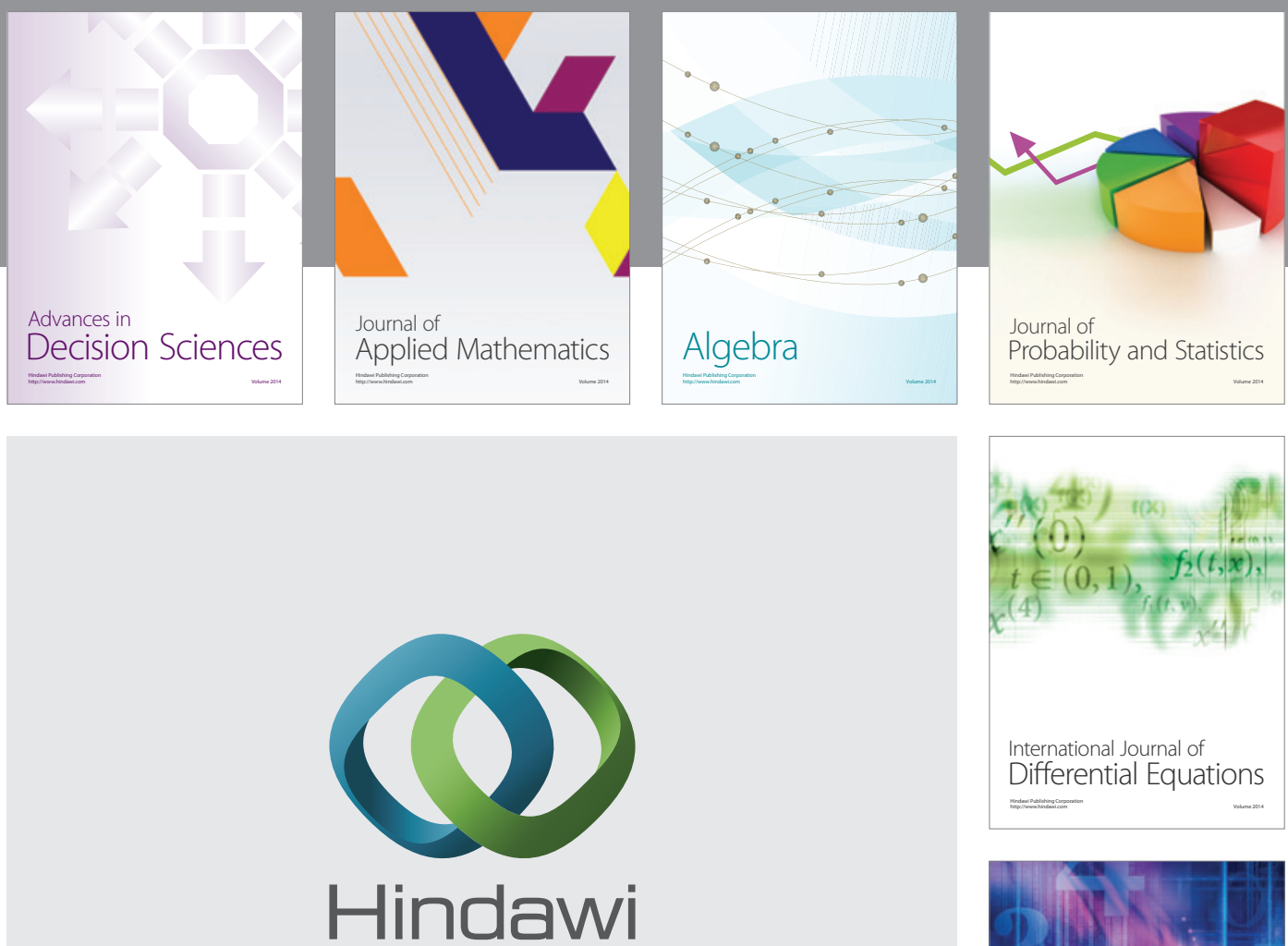

Submit your manuscripts at http://www.hindawi.com
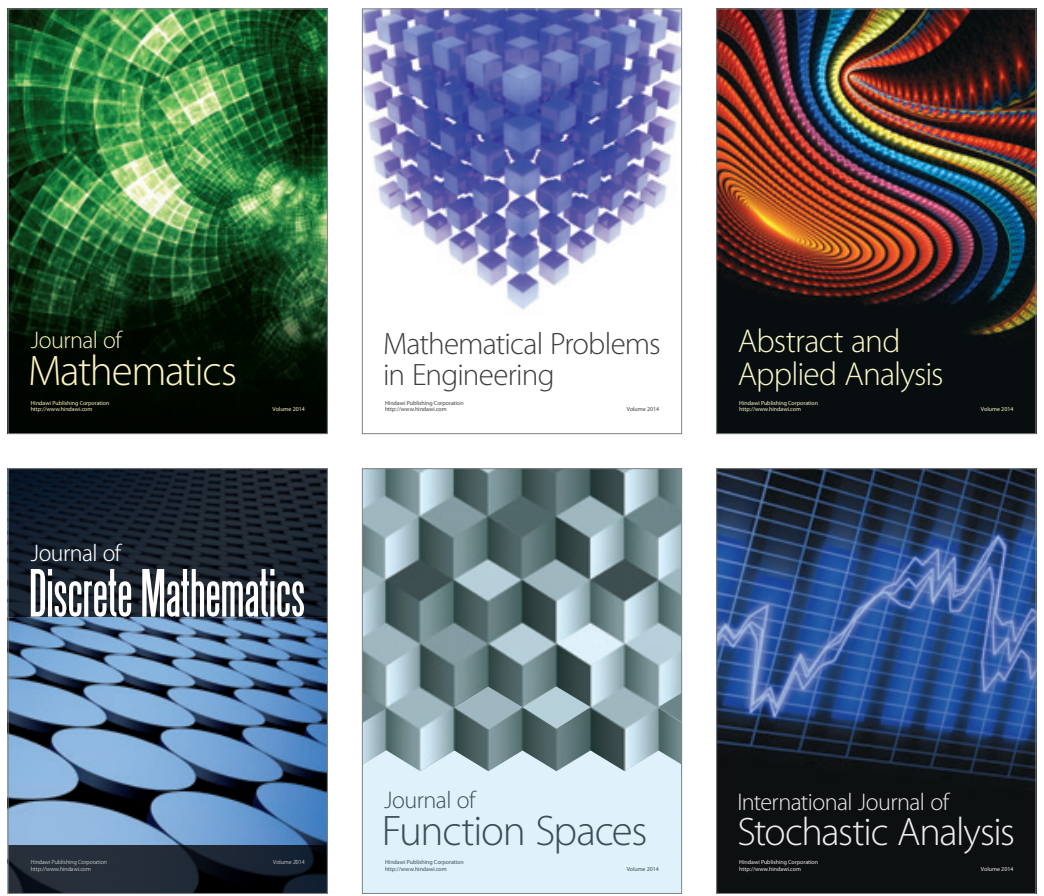

Journal of

Function Spaces

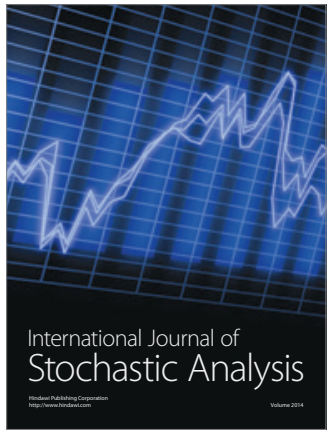

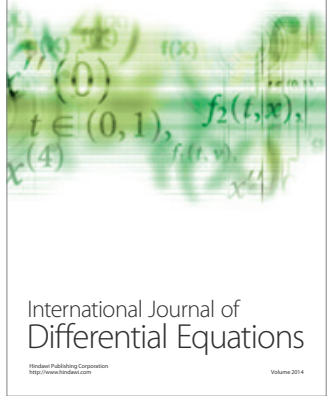
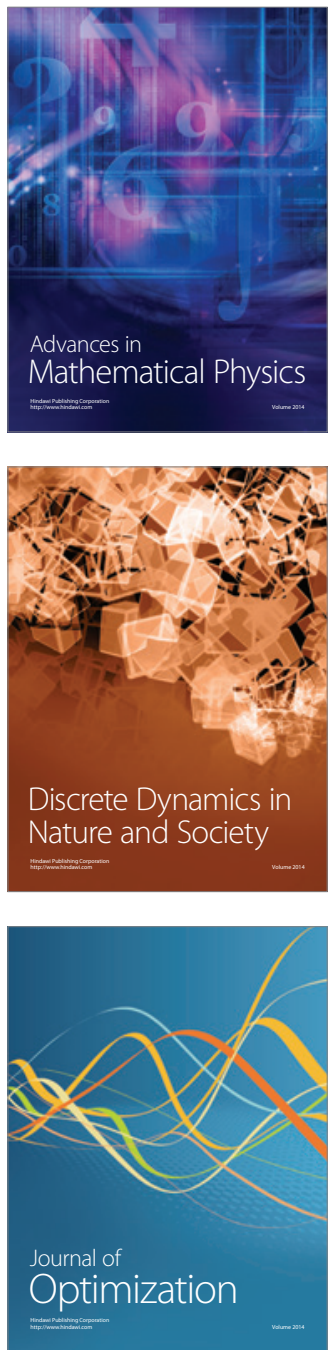